\title{
Clinical outcomes in patients treated with radiotherapy after surgery for cervical cancer
}

\author{
Kyungmi Yang, MD¹, Won Park, MD, PhD¹, Seung Jae Huh, MD, PhD¹, Duk-Soo Bae, MD, PhD², \\ Byoung-Gie Kim, MD, PhD'2, Jeong-Won Lee, MD, PhD² \\ Departments of ${ }^{1}$ Radiation Oncology and ${ }^{2}$ Obstetrics and Gynecology, Samsung Medical Center, \\ Sungkyunkwan University School of Medicine, Seoul, Korea
}

\begin{abstract}
Purpose: The purpose of this study was to analyze clinical outcomes from cervical cancer and stratify patients into risk groups for prognostic factors for early-stage disease.

Materials and Methods: We retrospectively reviewed patients with stage IB or IIA cervical cancer treated with adjuvant radiotherapy (RT) or concurrent chemoradiotherapy (CCRT) following primary surgery at Samsung Medical Center from 2001 to 2011. Adjuvant RT was added for patients with intermediate-risk factors, and adjuvant CCRT was performed on high-risk patients after surgery.

Results: We reviewed 247 patients-149 in the high-risk group and 98 in intermediate-risk group. The median follow-up was 62 months. Loco-regional failure (LRF) alone occurred in 7 patients (2.8\%), distant metastasis alone in 37 patients (15.0\%) and LRF with DM in 4 patients (1.6\%). The 5-year disease-free survival (DFS) and overall survival (OS) rates for both groups were $79.7 \%$ and $87.6 \%$, respectively. In the high-risk group, the 5-year DFS and OS probabilities were $72.5 \%$ and $81.9 \%$, respectively. Histologic type, pathologic tumor size, and the number of pelvic lymph node (PLN) metastasis were significant prognostic factors for DFS and OS. We suggest a scoring system (0-3) using these prognostic factors to predict poor prognosis in high-risk patients. Using this system, patients with higher scores have higher recurrence and lower survival rates.

Conclusion: In the high-risk cervical-cancer group who received primary surgery and adjuvant CCRT, non-squamous type, large tumor size and the number of PLN metastasis were significant prognostic factors, and the number of these factors was associated with survival rates.
\end{abstract}

Keywords: Uterine cervical neoplasms, Hysterectomy, Adjuvant radiotherapy, Adjuvant chemoradiotherapy

\section{Introduction}

Uterine cervical cancer is the fourth most common female cancer worldwide [1] and the sixth most frequently diagnosed cancer for women in Korea [2]. Incidence and mortality associated with cervical cancer have been decreasing continuously for several years, but the survival rate has not improved, despite advancements in diagnosis and treatment.

For staging and determining the best treatment approach for cervical cancer, most oncologists use the International Federation of Gynecology and Obstetrics (FIGO) clinical staging system [3]. Based on the FIGO staging system, a radical

Received 25 July 2016, Revised 7 September 2016, Accepted 21 September 2016.

Correspondence: Won Park, MD, PhD, Department of Radiation Oncology, Samsung Medical Center, Sungkyunkwan University School of Medicine, 81 Irwon-ro, Gangnam-gu, Seoul 06351, Korea. Tel: +82-2-3410-2601, Fax: +82-2-3410-2619, E-mail: wonro. park@samsung.com

(C) This is an Open Access article distributed under the terms of the Creative Commons Attribution Non-Commercial License (http://creativecommons.org/ licenses/by-nc/4.0/) which permits unrestricted non-commercial use, distribution, and reproduction in any medium, provided the original work is properly cited.

www.e-roj.org 
hysterectomy or definitive radiotherapy (RT) with or without chemotherapy may be recommended for patients with early stage (IB-IIA) cervical cancer. After primary radical surgery, adjuvant treatment is frequently indicated for early-stage patients who have poor prognostic risk factors to reduce the likelihood of recurrence and improve survival. Several studies have identified high-risk factors for recurrence and mortality, including parametrial invasion (PMI), pelvic lymph node (PLN) metastasis, and a positive resection margin (RM). For patients with any of these risk factors, adjuvant concurrent chemo-radiation therapy (CCRT) is often recommended [4]. Also, RT alone has been recommended as an adjuvant treatment for patients with intermediate risk factors, including lymphovascular invasion (LVI), deep stromal invasion, and large tumor size $[5,6]$.

The aim of this study was to analyze clinical outcomes from cervical cancer and stratify patients into risk groups for prognostic factors for early-stage disease.

\section{Materials and Methods}

We retrospectively reviewed FIGO stage IB or IIA cervical cancer patients treated with adjuvant RT or CCRT following primary surgery at Samsung Medical Center between 2001 and 2011. We excluded patients who: received neoadjuvant chemotherapy, did not undergo radical surgery, received adjuvant sequential chemo-radiation therapy, received $<45$ Gy of adjuvant RT or CCRT, or had small-cell carcinoma with surgery-confirmed metastasis to other organs or to at least one para-aortic lymph node (PALN). All patients were treated with a primary radical hysterectomy and pelvic lymphadenectomy, a removal of all lymph nodes from the external, internal, and common iliac vessels and the obturator fossa nodes, and/or PALN sampling. Adjuvant RT was added if there were positive pathologic results for two or more of the following intermediate-risk factors: LVI, invasion depth of at least half or tumor size $\geq 4 \mathrm{~cm}$ (intermediate-risk group). Adjuvant CCRT was performed if the patient was positive for one or more of the following high-risk factors: PMI, PLN metastasis, or positive RM (high-risk group).

Adjuvant RT was started within 4-6 weeks after surgery. External beam radiotherapy (EBRT) was administered to the entire pelvic region using a conventional four-field box technique. Radiation doses ranged from 45-50.4 Gy at 25-28 fractions and 5 fractions per week. Intracavitary brachytherapy was added after EBRT in patients with positive vaginal RMs and was delivered 3 times a week for a total of 18-24 Gy in
6 fractions. Concurrent chemotherapy regimens consisted of weekly cisplatin for 6 cycles or 5 -fluorouracil and cisplatin (FP) every 3 weeks for 2-3 cycles.

Follow-up treatment consisted of a physical examination, pap smear, and squamous-cell carcinoma antigen monitoring every 3 months after completing treatment for the first 2 years, and twice annually for the next 3 years. Imaging, including computed tomography, was performed once or twice every year. The follow-up period began after a radical hysterectomy was performed as the primary treatment. In case of recurrence, we classified failures by recurrent site as follows: local failure (LF) is defined as recurrence at vaginal stump, regional failure (RF) is PLN area or pelvic side wall recurrence without visceral organ metastasis, and distant metastasis (DM) is non-pelvic lymph node including PALN and visceral organ metastasis. Loco-regional failure (LRF) includes LF and RF.

For statistical analyses, we used Mann-Whitney $U$ test and Fisher's exact test for comparison of characteristics between CCRT and RT groups. Univariate and multivariate analyses were performed with base-line variables, high and intermediate risk factors, and the number of risk factors in each group. Kaplan-Meier survival curves to estimate disease-free survival (DFS) and overall survival (OS), and Cox proportional hazards regression models for multivariate analyses of prognostic factors, and we stratified high-risk group of patient by the number of these prognostic factors. A p-values of multiple tests were corrected using Bonferroni's method. A p-value < 0.05 was considered significant. We used IBM SPSS ver. 21 (IBM, Armonk, NY, USA).

\section{Results}

\section{Patient characteristics}

Patient characteristics are presented in Table 1. A total of 247 patients were reviewed. The median follow-up period was 62 months (range, 2 to 176 months) and the median age was 48 years (range, 23 to 75 years). Histology revealed that 75.3\% of the cases were squamous-cell carcinomas (SCC) and 84.6\% and $15.4 \%$ of cases were classified as FIGO stages IB and IIA, respectively.

Patients were divided into a high-risk group $(n=149)$ and an intermediate-risk group ( $n=98)$. In the high-risk group, SCC accounted for $74.5 \%$ of cases and $84.5 \%$ were FIGO stage IB, while $15.5 \%$ were IIA. Presence of high-risk factors based on surgical pathology included positive PMI (31.5\%), PLN metastasis (84.6\%), and positive RM (6.7\%). In 126 patients with PLN metastasis, 2 or more positive PLN were 84 (56.4\%). 
Table 1. Patients' characteristics

\begin{tabular}{|c|c|c|c|c|}
\hline & $\begin{array}{l}\text { All patients } \\
(n=247)\end{array}$ & $\begin{array}{l}\text { High-risk group } \\
(n=149)\end{array}$ & $\begin{array}{l}\text { Intermediate-risk group } \\
\qquad(\mathrm{n}=98)\end{array}$ & $p$-value ${ }^{a)}$ \\
\hline Follow-up (mo) & $62(2-176)$ & $59(2-169)$ & $67(2-176)$ & 0.420 \\
\hline Age (yr) & $48(23-75)$ & $48(24-72)$ & $48(23-75)$ & 0.057 \\
\hline \multicolumn{5}{|c|}{ Histologic cell type } \\
\hline $\mathrm{SCC}$ & $186(75.3)$ & $111(74.5)$ & $75(76.5)$ & \multirow[t]{3}{*}{0.936} \\
\hline$A D C$ & $53(21.5)$ & $33(22.1)$ & $20(20.4)$ & \\
\hline ASC & $8(3.2)$ & $5(3.4)$ & $3(3.2)$ & \\
\hline \multicolumn{5}{|l|}{ FIGO stage } \\
\hline $\mid \mathrm{B}$ & $209(84.6)$ & $126(84.5)$ & $83(84.7)$ & \multirow[t]{6}{*}{$0.999^{b)}$} \\
\hline |B1 & $149(60.3)$ & 85 (57.0) & $64(65.3)$ & \\
\hline IB2 & $60(24.3)$ & $41(27.5)$ & $19(19.4)$ & \\
\hline$\| A$ & $38(15.4)$ & $23(15.5)$ & $15(15.3)$ & \\
\hline$\| A 1$ & $24(9.7)$ & $15(10.1)$ & $9(9.2)$ & \\
\hline IIA2 & $14(5.7)$ & $8(5.4)$ & $6(6.1)$ & \\
\hline \multicolumn{5}{|l|}{ PMI } \\
\hline Negative & $200(81.0)$ & $102(68.5)$ & $98(100)$ & \multirow[t]{2}{*}{$\mathrm{N} / \mathrm{A}$} \\
\hline Positive & 47 (19.0) & 47 (31.5) & - & \\
\hline \multicolumn{5}{|l|}{ PLN metastasis } \\
\hline Negative & $121(49.0)$ & $23(15.4)$ & $98(100)$ & \multirow[t]{4}{*}{$\mathrm{N} / \mathrm{A}$} \\
\hline Positive & $126(51.0)$ & $126(84.6)$ & - & \\
\hline 1 & $42(17.0)$ & $42(28.2)$ & - & \\
\hline$\geq 2$ & $84(34.0)$ & $84(56.4)$ & - & \\
\hline \multicolumn{5}{|l|}{ RM } \\
\hline Negative & 237 (96.0) & 139 (93.3) & $98(100)$ & \multirow[t]{2}{*}{$N / A$} \\
\hline Positive & $10(4.0)$ & $10(6.7)$ & - & \\
\hline \multicolumn{5}{|c|}{ Depth of invasion } \\
\hline$<1 / 2$ & $8(3.2)$ & $5(3.4)$ & $3(3.1)$ & \multirow[t]{3}{*}{0.735} \\
\hline$\geq 1 / 2$ & 212 (85.8) & $117(78.5)$ & 95 (96.9) & \\
\hline Unreported & 27 (10.9) & 27 (18.1) & - & \\
\hline \multicolumn{5}{|l|}{ Tumor size (cm) } \\
\hline$<4$ & 107 (50.2) & $71(47.7)$ & 36 (36.7) & \multirow[t]{2}{*}{$<0.001$} \\
\hline$\geq 4$ & 140 (49.8) & 78 (52.3) & 62 (63.3) & \\
\hline \multicolumn{5}{|l|}{ LVI } \\
\hline Negative & $85(34.4)$ & 49 (32.9) & $43(43.9)$ & \multirow[t]{2}{*}{0.106} \\
\hline Positive & $162(65.6)$ & $100(67.1)$ & $55(56.1)$ & \\
\hline
\end{tabular}

Values are presented as median (range) or number (\%).

SCC, squamous cell carcinoma; ADC, adenocarcinoma; ASC, adenosquamous carcinoma; FIGO, International Federation of Gynecology and Obstetrics; PMI, parametrial invasion; PLN, pelvic lymph nodes; RM, resection margin; LVI, lymphovascular invasion.

${ }^{a)}$ Mann-Whitney U-test for comparison with follow-up and age, and Fisher exact test for comparison with other variables. ${ }^{b}$ Fisher exact test between high- and intermediate-risk group with FIGO stage (IB and IIA), not including IB1-2 or IIA1-2.

All patients in the high-risk group had one or more high-risk factors. Twenty-six patients (17.4\%) had two high risk factors and 4 patients $(2.7 \%)$ had all three factors.

The proportions of histologic cell types and clinical stages were similar between the intermediate-risk group and the high-risk group. Presence of intermediate-risk factors based on surgical pathology included stromal invasion depth $\geq 1 / 2$ (96.9\%), tumor size $\geq 4 \mathrm{~cm}$ (63.3\%), and positive LVI (56.1\%).
There were 82 patients (83.7\%) with two intermediate risk factors and 16 (16.3\%) with all factors.

\section{Patterns of failure}

During the follow-up periods, 48 patients (19.4\%) experienced recurrence and Table 2 shows the patterns of failure for both risk groups. In details, distribution of recurrence was as follows: LF 2, RF 4, LF and RF 1, DM without LRF 37, and 
Table 2. Patterns of failure

\begin{tabular}{lccc}
\hline Recurrence site & $\begin{array}{c}\text { All pa- } \\
\text { tients } \\
(\mathrm{n}=247)\end{array}$ & $\begin{array}{c}\text { High-risk } \\
\text { group } \\
(\mathrm{n}=149)\end{array}$ & $\begin{array}{c}\text { Intermediate-risk } \\
\text { group } \\
(\mathrm{n}=98)\end{array}$ \\
\hline Isolated LRF & $7(2.8)$ & $7(4.7)$ & - \\
DM without LRF & $37(15.0)$ & $28(18.8)$ & $9(9.2)$ \\
LRF + DM & $4(1.6)$ & $2(1.3)$ & $2(2.0)$ \\
Total & $48(19.4)$ & $37(24.8)$ & $11(11.2)$ \\
\hline
\end{tabular}

Values are presented as number (\%).

LRF, locoregional failure; DM, distant metastasis.
DM with LRF 4 patients. The most common site of DM was lung (16 patients), and no patients with lung metastasis had loco-regional failure. The second common DM was in PALN (13 patients), and there were 7 isolated PALN metastasis. The second common visceral DM was colorectal metastasis (6 patients).

In the high-risk group, recurrence occurred in 37 patients. Isolated LRF occurred in 7 patients (LF 2 RF 4, and LF and RF 1); DM without LRF occurred in 28 patients; and both LRF and DM occurred in 2 patients. In patients with DM, lung metastasis

Table 3. Univariate and multivariate analysis in the high-risk group

\begin{tabular}{|c|c|c|c|c|c|c|c|c|}
\hline \multirow{3}{*}{ High-risk group } & \multicolumn{4}{|c|}{ 5-yr disease-free survival } & \multicolumn{4}{|c|}{ 5-yr overall survival } \\
\hline & \multicolumn{2}{|c|}{ Univariate } & \multicolumn{2}{|l|}{ Multivariate } & \multicolumn{2}{|c|}{ Univariate } & \multicolumn{2}{|l|}{ Multivariate } \\
\hline & $\%$ & p-value & HR (95\% Cl) & p-value & $\%$ & p-value & $\mathrm{HR}(95 \% \mathrm{Cl})$ & p-value \\
\hline \multicolumn{9}{|l|}{ Age (yr) } \\
\hline$<50$ & 69.6 & 0.282 & $0.808(0.410-1.591)$ & 0.538 & 79.9 & 0.942 & $1.251(0.511-3.063)$ & 0.625 \\
\hline$\geq 50$ & 75.8 & & & & 84.5 & & & \\
\hline \multicolumn{9}{|l|}{ Histologic type } \\
\hline SCC & 78.0 & 0.016 & 2.033 (1.009-4.097) & 0.047 & 88.4 & 0.011 & $2.683(1.061-6.786)$ & 0.037 \\
\hline Non-SCC & 57.6 & & & & 64.6 & & & \\
\hline \multicolumn{9}{|l|}{ FIG0 stage } \\
\hline IB & 72.1 & 0.937 & $1.014(0.403-2.548)$ & 1.014 & 82.7 & 0.344 & $1.435(0.408-5.052)$ & 0.574 \\
\hline$\| A$ & 73.9 & & & & 78.3 & & & \\
\hline \multicolumn{9}{|l|}{ PMI } \\
\hline Negative & 70.8 & 0.557 & $0.650(0.251-1.683)$ & 0.375 & 82.8 & 0.987 & 1.212 (0.377-3.898) & 0.748 \\
\hline Positive & 76.6 & & & & 80.8 & & & \\
\hline \multicolumn{9}{|l|}{ PLN metastasis } \\
\hline Negative & 81.6 & 0.358 & $0.626(0.146-2.685)$ & 0.529 & 85.2 & 0.551 & $0.600(0.242-1.680)$ & 0.627 \\
\hline Positive & 70.8 & & & & 80.1 & & & \\
\hline $0-1$ & 80.6 & 0.027 & $2.197(0.933-5.174)$ & 0.072 & 91.0 & 0.073 & $2.518(0.773-8.200)$ & 0.125 \\
\hline$\geq 2$ & 65.8 & & & & 74.5 & & & \\
\hline \multicolumn{9}{|l|}{ RM } \\
\hline Negative & 73.8 & 0.215 & $1.822(0.564-5.885)$ & 0.316 & 83.2 & 0.215 & 1.209 (0.231-6.318) & 0.822 \\
\hline Positive & 54.0 & & & & 65.6 & & & \\
\hline \multicolumn{9}{|l|}{ No. of high risk factors ${ }^{a}$} \\
\hline 1 & 75.2 & 0.140 & $N / A$ & $N / A$ & 85.2 & 0.084 & N/A & N/A \\
\hline $2-3$ & 61.5 & & & & 69.4 & & & \\
\hline \multicolumn{9}{|l|}{ Depth of invasion } \\
\hline$<1 / 2$ & 100 & 0.240 & N/A & $N / A$ & 80.0 & 0.828 & $0.258(0.028-2.358)$ & 0.230 \\
\hline$\geq 1 / 2$ & 71.2 & & & & 82.7 & & & \\
\hline \multicolumn{9}{|l|}{ Tumor size $(\mathrm{cm})$} \\
\hline$<4$ & 83.6 & 0.008 & $2.086(0.972-4.476)$ & 0.059 & 88.0 & 0.056 & $3.462(1.015-11.814)$ & 0.047 \\
\hline$\geq 4$ & 63.1 & & & & 66.7 & & & \\
\hline \multicolumn{9}{|l|}{ LVI } \\
\hline Negative & 72.0 & 0.656 & $1.121(0.528-2.380)$ & 0.766 & 78.6 & 0.508 & 0.638 (0.242-1.680) & 0.363 \\
\hline Positive & 72.8 & & & & 83.6 & & & \\
\hline
\end{tabular}

$\mathrm{HR}$, hazard ratio; $\mathrm{Cl}$, confidence interval; SCC, squamous cell carcinoma; FIGO, International Federation of Gynecology and Obstetrics; $\mathrm{PMI}$, parametrial invasion; PLN, pelvic lymph node; RM, resection margin; LVI, Iymphovascular invasion; N/A, not available.

${ }^{a}$ The number of positive high risk factors including PMI, PLN metastasis, and RM. 
was the most common site of DM (11 patients), and PALN metastasis was the second incidence of DM (10 patients), and 6 were isolated without other metastasis. Supraclavicular lymph node $(\mathrm{SCL})$ metastasis was in 6 patients and 4 were isolated. Other sites of DM were in colorectum (3 patients) and pelvic bone (2 patients).

In the intermediate-risk group, recurrence occurred in 11 patients. DM without LRF occurred in 9 patients and both LRF and DM occurred in 2 patients, while isolated LRF did not occur in any of our study patients. Distribution of DM was similar with CCRT group; lung 5 patients, PALN 3 patients, and colorectum 3 patients.

A

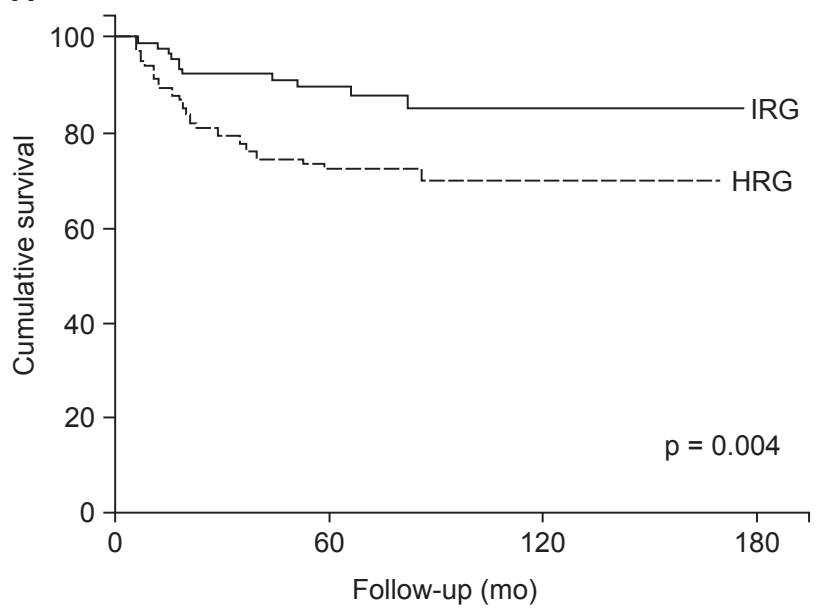

\section{Survival outcomes and prognostic factors}

Our survival analyses of all patients found that the 5 -year DFS probability was $79.7 \%$ and the 5 -year overall survival (OS) probability was $87.6 \%$.

After separating patients into high- and intermediate-risk groups, the 5-year DFS probabilities were $72.5 \%$ and $90.0 \%$ $(p=0.004)$, respectively, and the 5 -year 0 s probabilities were $81.9 \%$ and $95.7 \%$ ( $p=0.002$ ) (Fig. 1). According to univariate and multivariate analyses, histologic type, tumor size, and the number of positive PLN were associated with DFS or OS in the high-risk group (Table 3). Non-SCC type was a significant prognostic factor for predicting DFS and OS in multivariate

B

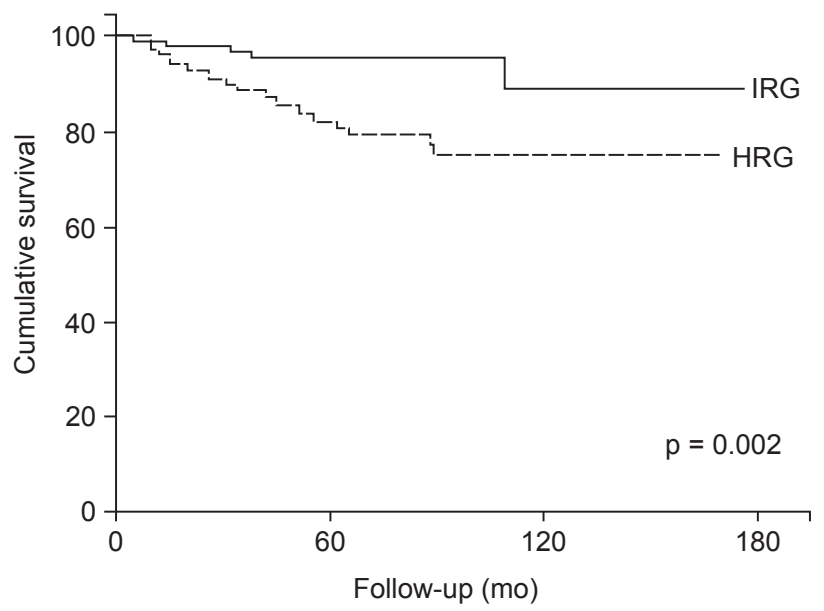

Fig. 1. (A) Disease-free survival and (B) overall survival in the high-risk group (HRG) and intermediate-risk group (IRG).

A

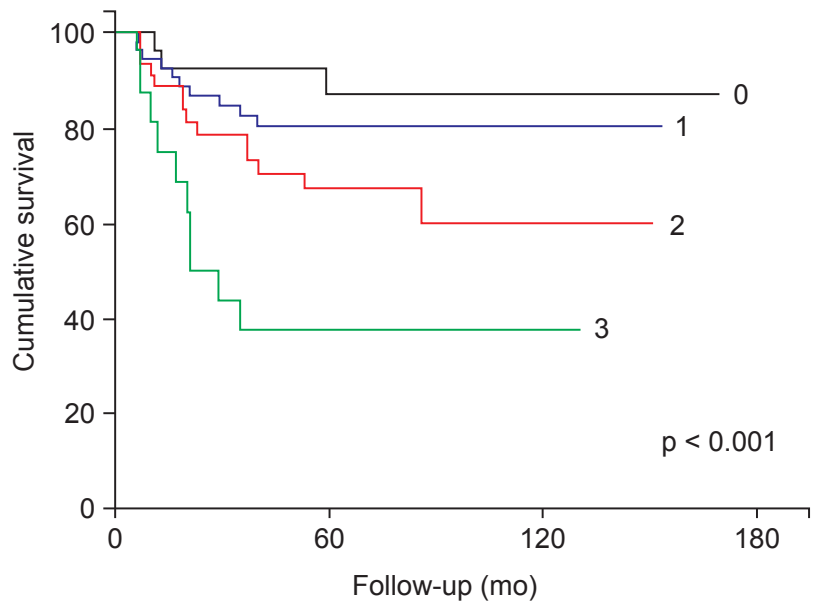

B

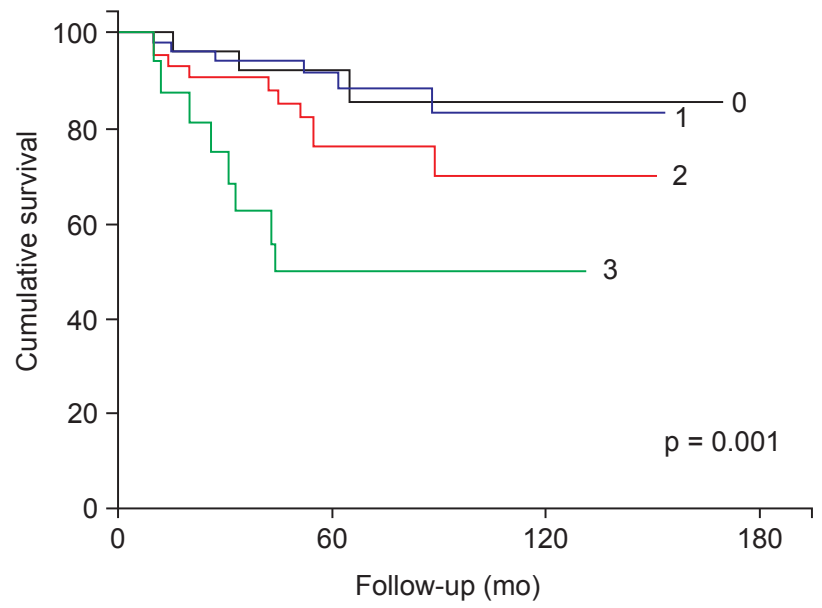

Fig. 2. (A) Disease-free survival and (B) overall survival of subgroups stratified by prognostic risk score (the score means the number of positive factors including non-squamous cell carcinoma type, pathologic tumor size $\geq 4 \mathrm{~cm}$, and pelvic lymph node metastasis $\geq 2$ ). 
Table 4. Univariate and multivariate analysis in the intermediate-risk group

\begin{tabular}{|c|c|c|c|c|c|c|c|c|}
\hline \multirow{3}{*}{ Intermediate-risk group } & \multicolumn{4}{|c|}{ 5-yr disease-free survival } & \multicolumn{4}{|c|}{ 5-yr overall survival } \\
\hline & \multicolumn{2}{|c|}{ Univariate } & \multicolumn{2}{|l|}{ Multivariate } & \multicolumn{2}{|c|}{ Univariate } & \multicolumn{2}{|l|}{ Multivariate } \\
\hline & $\%$ & p-value & HR (95\% Cl) & $p$-value & $\%$ & p-value & HR (95\% Cl) & p-value \\
\hline \multicolumn{9}{|l|}{ Age (yr) } \\
\hline$<50$ & 86.1 & 0.256 & $0.459(0.116-1.819)$ & 0.268 & 94.1 & 0.292 & $0.471(0.048-4.620)$ & 0.518 \\
\hline$\geq 50$ & 95.1 & & & & 97.7 & & & \\
\hline \multicolumn{9}{|l|}{ Histologic type } \\
\hline SCC & 92.6 & 0.117 & 2.599 (0.759-8.901) & 0.128 & 97.2 & 0.088 & $3.587(0.545-23.626)$ & 0.184 \\
\hline Non-SCC & 82.1 & & & & 91.1 & & & \\
\hline \multicolumn{9}{|l|}{ FIGO stage } \\
\hline IB & 89.5 & 0.692 & $0.914(0.163-5.145)$ & 0.919 & 97.0 & 0.401 & 1.409 (0.199-10.001) & 0.732 \\
\hline$\| A$ & 91.7 & & & & 91.8 & & & \\
\hline \multicolumn{9}{|l|}{ Depth of invasion } \\
\hline$<1 / 2$ & 100 & 0.485 & $N / A$ & $N / A$ & 100 & 0.712 & $N / A$ & $\mathrm{~N} / \mathrm{A}$ \\
\hline$\geq 1 / 2$ & 89.6 & & & & 95.5 & & & \\
\hline \multicolumn{9}{|l|}{ Tumor size $(\mathrm{cm})$} \\
\hline$<4$ & 84.5 & 0.218 & $0.238(0.025-2.268)$ & 0.212 & 96.9 & 0.419 & $1.167(0.059-22.964)$ & 0.919 \\
\hline$\geq 4$ & 93.3 & & & & 95.0 & & & \\
\hline \multicolumn{9}{|l|}{ LVI } \\
\hline Negative & 90.2 & 0.715 & $0.466(0.051-4.262)$ & 0.499 & 92.7 & 0.452 & $0.596(0.058-6.093)$ & 0.662 \\
\hline Positive & 89.9 & & & & 98.0 & & & \\
\hline \multicolumn{9}{|c|}{ No. of intermediate risk factors ${ }^{a)}$} \\
\hline 2 & 88.0 & 0.470 & N/A & $N / A$ & 94.8 & 0.834 & $N / A$ & N/A \\
\hline 3 & 100 & & & & 100 & & & \\
\hline
\end{tabular}

$\mathrm{HR}$, hazard ratio; $\mathrm{Cl}$, confidence interval; SCC, squamous cell carcinoma; FIGO, International Federation of Gynecology and Obstetrics; LVI, Iymphovascular invasion; N/A, not available.

${ }^{a}$ The number of positive intermediate risk factors including depth of invasion $\geq 1 / 2$, tumor size $\geq 4 \mathrm{~cm}$, and lymphovascular invasion.

analyses ( $p=0.047$ and $p=0.037$, respectively). Large tumor size was a significant factor for predicting OS in multivariate analyses ( $p=0.047)$, but not for DFS ( $p=0.059)$. Well-known risk factors, including PMI, PLN metastasis, and positive RM, were not significant for any of our survival analyses. The number of positive PLN, however, had a trend of difference with DFS ( $p=0.072)$. When we considered the number of high risk factors including PMI, PLN metastasis and positive RM, we found that patients with two or more positive high risk factors had lower DFS and OS than patients with only one risk factor, although the trend was not significant.

In the intermediate-risk group, none of the factors, including large tumor size and LVI, were associated with DFS and $O S$ in univariate and multivariate analyses (Table 4). We were not able to analyze the association between deep stromal invasion and survival rates in multivariate analyses because none of the patients who did not have deep stromal invasion experienced a recurrence or were censored. Furthermore, we found that the number of intermediate risk factors a patient had was not associated with survival.

\section{Prognostic stratification for the high-risk patients}

As previously stated, histologic cell type, tumor size and the number of PLN metastasis were prognostic factors for the high-risk group. Thus, we developed a scoring system to identify the patients in the high-risk group who had higher recurrence rate and poorer prognosis (Table 5). Our prognostic risk score incorporates prognostic factors such as non-SCC type (1 point), tumor size $\geq 4 \mathrm{~cm}$ ( 1 point), and 2 or more PLN metastasis (1 point). Subgroups of patients based on prognostic risk scores had significantly different DFS and OS rates (Fig. 2). The 5-year DFS probabilities for patients with scores of $0-1$ and $2-3$ were $82.6 \%$ and $58.9 \%$, respectively ( $p$ $=0.001) ;$ OS probabilities were $91.9 \%$ and $68.8 \%$, respectively ( $p$ $=0.005$ ).

\section{Discussion and Conclusion}

We investigated outcomes and prognostic factors for earlystage cervical-cancer patients who received adjuvant therapy following primary surgery. Adjuvant RT is an additional 
Table 5. Survival analysis with prognostic risk scoring in the high-risk group

\begin{tabular}{|c|c|c|c|c|c|c|c|c|c|c|}
\hline \multirow{3}{*}{ Prognostic risk score ${ }^{a)}$} & \multicolumn{5}{|c|}{ 5-yr disease-free survival } & \multicolumn{5}{|c|}{ 5-yr overall survival } \\
\hline & \multirow{2}{*}{$\%$} & \multicolumn{4}{|c|}{ p-value } & \multirow{2}{*}{$\%$} & \multicolumn{4}{|c|}{$p$-value } \\
\hline & & $A \|^{b)}$ & $1^{c)}$ & $2^{\text {c) }}$ & $3^{\text {c) }}$ & & $A l^{b)}$ & $1^{c)}$ & $2^{c)}$ & $3^{c)}$ \\
\hline $0(n=28)$ & 87.1 & $<0.001$ & 0.999 & 0.138 & $<0.001$ & 92.3 & 0.001 & 0.999 & 0.687 & 0.012 \\
\hline $1(n=58)$ & 80.5 & & - & 0.390 & $<0.001$ & 91.7 & & - & 0.372 & $<0.001$ \\
\hline $2(n=47)$ & 67.4 & & - & - & 0.087 & 76.4 & & - & - & 0.111 \\
\hline $3(n=16)$ & 37.5 & & - & - & - & 50.0 & & - & - & - \\
\hline $0-1(n=86)$ & 82.6 & ค $\cap \cap \cap 1$ & & & & 91.9 & & & & \\
\hline $2-3(n=63)$ & 58.9 & 0.001 & & & & 68.8 & 0.005 & & & \\
\hline
\end{tabular}

SCC, squamous cell carcinoma.

${ }^{\text {a) }}$ The number of positive factors including non-SCC type, pathologic tumor size $\geq 4 \mathrm{~cm}$, and pelvic lymph node metastasis $\geq 2$. ${ }^{\text {b) }} \mathrm{Ka}-$ plan-Meier survival analysis with all groups. ${ }^{c}$ Kaplan-Meier survival analysis between each group and p-values of multiple tests were corrected by Bonferroni method.

modality to improve local control and overall survival in patients with a poor prognosis based on surgical pathologic findings [4-7]. In our study, the loco-regional control rate was over 90\% after adjuvant RT or CCRT following primary surgery and DM accounted for the majority of treatment failures. The recurrence rate was $24.8 \%$ in the high-risk group and $11.2 \%$ in the intermediate-risk group.

We analyzed each group of patients to determine the relationships between risk factors and survival outcomes. In the intermediate-risk group, we did not find any significant relationships between risk factors and survival outcomes. In the high-risk group, however, histologic cell type, pathologic tumor size, and the number of PLN metastasis were prognostic factors associated with DFS or OS, while other high-risk factors, such as PMI, PLN metastasis, and positive RM, were not associated with survival differences. With histologic cell type, some retrospective studies have found that histologic subtype in early-stage cervical cancer impacts survival [7-10]. Adenocarcinoma was associated with a worse prognosis than SCC type in patients with risk factors identified during surgical pathology [11], whereas other studies found no difference in survival between the two histologic types or reports found survival differences between histologic types among stageII cases, but not among stage-I cases [12-14]. Also, tumor size was found to only affect survival in the high-risk group. Some studies reported that large tumor size was a poor predictor of recurrence and mortality but did have an effect on increases in PLN metastasis $[7,15]$. Recent guidelines recommend a definitive CCRT for patients with clinically large tumors (stage IB2 or IIA2). For early-stage patients treated with primary surgery, adjuvant RT alone can be added if two or more positive risk factors are present, including a pathologically large tumor, while patients identified as having any high-risk factors by surgical pathology are recommended to undergo CCRT regardless of tumor size. In this study, however, we found that tumor size affected recurrence and survival, even in the high-risk group. Lymph node metastasis is known as a risk factor of cervical cancer [4], although it does not modify the FIGO clinical stage. In this study, however, PLN metastasis was not significant with survival. Instead of lymph node status, the number of positive PLN metastasis was associated with DFS in univariate analyses. The effect of the number of PLN metastasis has not to be determined, but previous studies reported that there was no difference between survivals of patients with 0 and those with 1 positive node [16-18] and the number of positive lymph nodes might be considerable a prognostic factor in stage IB-IIB cervical cancer patients [19] These prognostic factors we found in this study should be considered as an additional or secondary risk factor, although these need to be definitively validated in a follow-up study.

Stratification by number of present intermediate-risk factors has been used to determine the likelihood of receiving adjuvant RT [5]; however, few studies have explored the same relationships with high-risk factors. Previous studies of risk factors and treatments did not discuss whether the number of risk factors was an important indicator [4]. We found that an increase in the number of high-risk factors was associated with an increase in likelihood of recurrence and a decrease in survival, although these trends were not statistically significant. Patients in this study were not distributed evenly within their risk groups. Among patients in the high-risk group, $84.6 \%$ had PLN metastasis, while a much smaller proportion had recurrent cases with positive RM than other groups with PLN metastasis and PMI. Thus, further evaluations 
from a well-designed study and a large sample size are needed to assess whether risk stratification by number of risk factors reveals important prognostic factors for high-risk patients.

We focused our risk stratifications on patients with existing or additional prognostic factors, and observed that patients with non-SCC, large tumors, and 2 or more PLN metastasis had poorer prognosis than other participants in our study. Although poorer prognosis in patients with certain conditions, such as large tumor size, adenocarcinoma, or positive PLN metastasis after primary surgery, have been reported $[4,7]$, there are no confirmed criteria for determining which patients need additional treatment; nor is there enough evidence to alter recent treatment guidelines for high-risk patients. Nevertheless, previous studies of patents with locally advanced cervical cancer, from our center and others, have consistently reported that consolidation chemotherapy after primary CCRT might enhance local control and eradicate distant micrometastases [20-23]. A phase-II study that enrolled high-risk early-stage cervical cancer patients as well as locally advanced patients concluded that consolidation chemotherapy had low efficacy [24]. Meanwhile, a recent phase-II study suggested that pelvic CCRT with intensity-modulated radiotherapy followed by consolidation chemotherapy might be highly effective in surgically treated cervical-cancer patients with positive PLN; 3-year progression-free survival and OS among these patients were $88.5 \%$ and $93.8 \%$, respectively, and these outcomes are superior to those from historical control groups who received adjuvant CCRT without consolidation [25]. However, there has been no consensus on regimens for consolidation chemotherapy or even adjuvant CCRT. Most patients in our study and in historical control groups were treated with platinum-based chemotherapy, while other highly cited studies used carboplatin plus paclitaxel for CCRT or consolidation chemotherapy. Furthermore, long-term chemotherapy might cause more severe or higher frequency toxicity $[24,25]$. Also, there have been no randomized trials evaluating consolidation chemotherapy following adjuvant CCRT.

Recently, some randomized trials for more aggressive adjuvant treatment to improve clinical outcomes are ongoing: RTOG 0724, one of phase-III trials considering adjuvant CCRT with or without additional chemotherapy in high-risk patients [26], and GOG 0263, a phase-III trial of adjuvant RT with or without concurrent chemotherapy in intermediate-risk patients [27]. We expect that worthy findings from them will be helpful to decide optimized postoperative treatment plan for cervical cancer patients.
This retrospective study had several limitations. We included cervical-cancer patients treated with adjuvant treatment following a primary radical surgery; thus, a selection bias was present. Also, there was not a uniform protocol for adjuvant chemotherapy regimens in our study. Finally, some of our patient subgroups were very small, which limited our ability to statistically evaluate the effect of the number of risk factors and to determine the significance of additional prognostic factors.

In conclusion, in this study, non-SCC type, large tumor size, and 2 or more PLN metastasis were significant prognostic factors among high-risk patients with early-stage cervical cancer who were treated with radical surgery and adjuvant CCRT. Also, we suspect that the number of these prognostic factors is associated with recurrence and survival.

\section{Conflict of Interest}

No potential conflict of interest relevant to this article was reported.

\section{References}

1. Torre LA, Bray F, Siegel $R L$, Ferlay J, Lortet-Tieulent J, Jemal $A$. Global cancer statistics, 2012. CA Cancer J Clin 2015;65:87108.

2. Jung KW, Won YJ, Kong HJ, et al. Cancer statistics in Korea: incidence, mortality, survival, and prevalence in 2012. Cancer Res Treat 2015;47:127-41.

3. Pecorelli S, Zigliani L, Odicino F. Revised FIGO staging for carcinoma of the cervix. Int J Gynaecol Obstet 2009;105:107-8.

4. Peters WA 3rd, Liu PY, Barrett RJ 2nd, et al. Concurrent chemotherapy and pelvic radiation therapy compared with pelvic radiation therapy alone as adjuvant therapy after radical surgery in high-risk early-stage cancer of the cervix. J Clin Oncol 2000;18:1606-13.

5. Rotman M, Sedlis A, Piedmonte MR, et al. A phase III randomized trial of postoperative pelvic irradiation in Stage IB cervical carcinoma with poor prognostic features: follow-up of a gynecologic oncology group study. Int J Radiat Oncol Biol Phys 2006;65:169-76.

6. Sedlis A, Bundy BN, Rotman MZ, Lentz SS, Muderspach LI, Zaino RJ. A randomized trial of pelvic radiation therapy versus no further therapy in selected patients with stage IB carcinoma of the cervix after radical hysterectomy and pelvic Iymphadenectomy: a Gynecologic Oncology Group Study. Gynecol Oncol 1999;73:177-83.

7. Kim HJ, Rhee WJ, Choi SH, et al. Clinical outcomes of adjuvant radiation therapy and prognostic factors in early stage uterine 
cervical cancer. Radiat Oncol J 2015;33:126-33.

8. Waggoner SE. Cervical cancer. Lancet 2003;361:2217-25.

9. Lee YY, Choi CH, Kim TJ, et al. A comparison of pure adenocarcinoma and squamous cell carcinoma of the cervix after radical hysterectomy in stage IB-IIA. Gynecol Oncol 2011;120:439-43.

10. Noh JM, Park W, Kim YS, et al. Comparison of clinical outcomes of adenocarcinoma and adenosquamous carcinoma in uterine cervical cancer patients receiving surgical resection followed by radiotherapy: a multicenter retrospective study (KROG 13-10). Gynecol Oncol 2014;132:618-23.

11. Mabuchi S, Okazawa M, Matsuo K, et al. Impact of histological subtype on survival of patients with surgically-treated stage IA2-IIB cervical cancer: adenocarcinoma versus squamous cell carcinoma. Gynecol Oncol 2012;127:114-20.

12. Landoni $F$, Maneo $A$, Colombo $A$, et al. Randomised study of radical surgery versus radiotherapy for stage Ib-lla cervical cancer. Lancet 1997;350:535-40.

13. Huang YT, Wang CC, Tsai CS, et al. Clinical behaviors and outcomes for adenocarcinoma or adenosquamous carcinoma of cervix treated by radical hysterectomy and adjuvant radiotherapy or chemoradiotherapy. Int J Radiat Oncol Biol Phys 2012;84:420-7.

14. Grisaru D, Covens A, Chapman B, et al. Does histology influence prognosis in patients with early-stage cervical carcinoma? Cancer 2001;92:2999-3004.

15. Chung $C K$, Nahhas WA, Stryker JA, Curry SL, Abt AB, Mortel R. Analysis of factors contributing to treatment failures in stages IB and IIA carcinoma of the cervix. Am J Obstet Gynecol 1980;138:550-6.

16. Lai $\mathrm{CH}$, Chang HC, Chang TC, Hsueh S, Tang SG. Prognostic factors and impacts of adjuvant therapy in early-stage cervical carcinoma with pelvic node metastases. Gynecol Oncol 1993:51:390-6.

17. Park TK, Kwon JY, Kim SW, Kim SH, Kim SN, Kim GE. Patterns of treatment failure following radiotherapy with combination chemotherapy for patients with high-risk stage IIB cervical carcinoma. Int J Clin Oncol 2004;9:120-4.

18. Sakuragi N, Satoh C, Takeda N, et al. Incidence and distribution pattern of pelvic and paraaortic lymph node metastasis in patients with Stages IB, IIA, and IIB cervical carcinoma treated with radical hysterectomy. Cancer 1999;85:1547-54.

19. Inoue $T$, Morita K. The prognostic significance of number of positive nodes in cervical carcinoma stages IB, IIA, and IIB. Cancer 1990;65:1923-7.

20. Choi CH, Lee JW, Kim TJ, et al. Phase II study of consolidation chemotherapy after concurrent chemoradiation in cervical cancer: preliminary results. Int J Radiat Oncol Biol Phys 2007;68:817-22.

21. Zhang MO, Liu SP, Wang XE. Concurrent chemoradiotherapy with paclitaxel and nedaplatin followed by consolidation chemotherapy in locally advanced squamous cell carcinoma of the uterine cervix: preliminary results of a phase II study. Int J Radiat Oncol Biol Phys 2010;78:821-7.

22. Abe $A$, Furumoto $H$, Nishimura $M$, Irahara $M$, Ikushima H. Adjuvant chemotherapy following concurrent chemoradiotherapy for uterine cervical cancer with Iymphadenopathy. Oncol Lett 2012;3:571-6.

23. Petric Mise B, Boraska Jelavic T, Strikic $A$, et al. Long followup of patients with locally advanced cervical cancer treated with concomitant chemobrachyradiotherapy with cisplatin and ifosfamide followed by consolidation chemotherapy. Int J Gynecol Cancer 2015;25:315-9.

24. Kim HS, Kim MK, Kim HJ, Han SS, Kim JW. Phase II study of consolidation chemotherapy after adjuvant or primary concurrent chemoradiation using paclitaxel and carboplatin to treat high-risk early-stage or locally advanced cervical cancer. Cancer Res Treat 2012;44:97-103.

25. Mabuchi S, Isohashi F, Yokoi T, et al. A phase II study of postoperative concurrent carboplatin and paclitaxel combined with intensity-modulated pelvic radiotherapy followed by consolidation chemotherapy in surgically treated cervica cancer patients with positive pelvic lymph nodes. Gynecol Oncol 2016;141:240-6.

26. ClinicalTrials.gov. Trial of postoperative chemoradiotherapy with or without consolidation chemotherapy for cervical cancer patients (NCT01755845) [Internet]. Bethesda, MD: National Library of Medicine (US); c2016 [cited 2016 May 1]. Available from: https://clinicaltrials.gov/ct2/show/NCT017558 45?term $=01755845$ \&trank=1.

27. ClinicalTrials.gov. Radiation therapy with or without chemotherapy in patients with stage I or stage II cervical cancer who previously underwent surgery (NCT01101451) [Internet]. Bethesda, MD: National Library of Medicine (US); c2016 [cited 2016 May 1]. Available from: https://clinicaltrials. gov/show/NCT01101451. 